\title{
TIPOS DE PREENSÃO E MOVIMENTOS DO PUNHO DURANTE ATIVIDADE DE MANUSEIO DE CARGA
}

\author{
Padula RS ${ }^{1,2}$, Souza VC ${ }^{1}$, Gil Coury HJC ${ }^{1}$ \\ ${ }^{1}$ Departamento de Fisioterapia, Universidade Federal de São Carlos, São Carlos, SP \\ ${ }^{2}$ Curso de Fisioterapia, Universidade São Francisco, Bragança Paulista, SP \\ Correspondência para: Rosimeire Simprini Padula, Av. Atibaia, 235, Apto 4, Atibaia Jardim, CEP 12942-170, Atibaia, SP, \\ e-mail: padula@iris.ufscar.br
}

Recebido: 16/09/2004 - Aceito: 02/02/2005

\begin{abstract}
RESUMO
Introdução: O tipo de tarefa realizada determina o tipo de preensão utilizada, a força aplicada, bem como as posturas adotadas para sua execução. Diferentes posturas e preensões envolvidas nessas tarefas apresentam maiores ou menores riscos de lesões musculoesqueléticas. Objetivo: Descrever os tipos de preensão empregados durante atividades de manuseio de cargas de 5 e $10 \mathrm{~kg}$ entre superfícies de alturas diferentes e, quantificar os movimentos de flexo/extensão, desvio radial e ulnar do punho registrados por meio da eletrogoniometria. Método: 10 voluntários masculinos manusearam caixa entre superfícies de alturas diferentes. Os tipos de preensão utilizados foram filmados. Diferentes categorias de preensão utilizadas durante essa tarefa foram identificadas. As amplitudes de movimentos e a porcentagem de tempo gasto dentro e fora das faixas de amplitudes préestabelecidas ( $15^{0}$ de flexão e extensão, $10^{\circ}$ de desvio radial, e $15^{0}$ desvio ulnar) foram determinadas. Uma análise de univariância foi utilizada para avaliar diferenças significativas entre a antropometria das mãos dos voluntários, e entre os movimentos utilizados para o manuseio de cargas e alturas diferentes. Resultados e Conclusões: Os resultados mostraram que as alturas das superfícies para as quais os objetos eram manuseados influenciaram significativamente $(\mathrm{p}=0,000)$ as amplitudes articulares, contudo não houve diferença nos movimentos para as diferentes massas das cargas manuseadas $(p=0,43)$. Tempo acima do considerado recomendável foi gasto em desvio radial quando os manuseios envolviam superfícies altas. Recomendações sobre local adequado para o acondicionamento de material a ser manuseado deveriam constar nos programas de treinamento visando a prevenção de lesões musculoesqueléticas.
\end{abstract}

Palavras-chave: preensão, manuseio de carga, disfunções musculoesqueléticas, eletrogoniometria.

\section{ABSTRACT}

\section{Types of Grip and Wrist Movements During Load Handling Activities}

Background: The type of task performed determines the type of grip utilized, the force applied and the postures adopted for carrying out the task. The different postures and grips involved in these tasks present higher or lower risks of musculoskeletal injuries. Objective: To describe the types of grip utilized when transferring 5 and $10 \mathrm{~kg}$ loads between surfaces at different heights, and to quantify the flexion/extension movements and the radial and ulnar deviations presented by the wrist, by means of recordings on an electrogoniometer. Method: Ten male volunteers transferred a box between surfaces at different heights. The types of grip utilized were videotaped. Different categories of grip utilized during this task were identified. The movement amplitudes and the percentages of time spent within and outside of preestablished amplitude ranges $\left(0-15^{\circ}\right.$ flexion/extension, $0-10^{\circ}$ radial deviation and $0-10^{\circ}$ ulnar deviation) were determined. Univariate analysis was utilized to evaluate any significant differences between the volunteers' hand dimensions and between the movements utilized for load-handling relating to different heights. Results and Conclusions: The results showed that the heights of the surfaces to which the objects were transferred significantly influenced the joint movement amplitudes $(\mathrm{p}=0.000)$. However, there was no difference between movements for the different loads handled ( $\mathrm{p}=0.43)$. A time greater that what is considered recommendable was spent on radial deviation when the handling activity involved high surfaces. Recommendations on appropriate locations for packing materials that are to be handled should be included in training programs aimed at preventing musculoskeletal injuries.

Key words: grip, load handling, musculoskeletal disorders, electrogoniometry. 


\section{INTRODUÇÃO}

O uso das mãos está presente num grande número de atividades realizadas pelo homem, desde aquelas relacionadas à vida diária, até as de ordem ocupacional e recreativa. Assim, além de ter uma importância no que se refere às atividades vitais de um indivíduo, o uso da mão pode ser considerado como um meio de integração social.

Vários estudos têm sido realizados para avaliar a incidência das disfunções musculoesqueléticas relacionadas às atividades de manuseio ${ }^{1,2}$. O que tem sido observado é que as atividades de manuseio afetam principalmente os membros superiores e tronco. No entanto, apesar de existirem muitos estudos avaliando a sobrecarga musculoesquelética presente nas tarefas de manuseio de cargas e seu efeito para a coluna vertebral, muito pouco tem sido estudado sobre os efeitos dessa tarefa para o punho. Vários parecem ser os fatores que influenciam a ocorrência das lesões durante o manuseio, dentre os quais, o peso e o tipo da carga manuseada ${ }^{1}$.

Ilda $^{3}$ relata que o formato da carga é uma condição que influi na preensão utilizada, pois objetos cujos formatos se aproximam da anatomia das mãos, proporcionam maior contato destas com o objeto permitindo maior firmeza de preensão. Nesse caso, uma quantidade menor de força é utilizada, facilitando assim, o manuseio. Enquanto que, um objeto maior necessita de mais força para mantê-lo e um número maior de segmentos corporais são recrutados para estabilizá-lo.

Um outro fator importante para a preensão é a posição das mãos e punhos, pois a força gerada na preensão é maior quando a mão está em posição neutra ou em pequena extensão, e é reduzida quando o punho está flexionado ou lateralizado. O desvio ulnar resulta em uma perda de $25 \%$ da força de preensão, e o desvio radial pode estar associado a uma perda de $20 \%$ da força ${ }^{4}$. As características individuais do sujeito devem também ser consideradas quando se analisa o tipo de preensão, pois o tamanho das mãos irá induzir o tipo de preensão a ser adotado, com isso variações na forma e dimensões da mão interferem na localização, direção e distribuição das forças envolvidas na preensão ${ }^{5}$.

Os estudos da preensão são mais realizados para avaliar a eficácia do redesenho de ferramentas de trabalho, e para diminuir os riscos de lesão musculoesqueléticas ${ }^{6}$. Contudo, a preensão empregada para segurar objetos não se restringe apenas ao manuseio de ferramentas, outros objetos são manuseados diariamente, em contextos ocupacionais, tais como, caixas, cilindros, carrinhos, sendo considerados também atividade de grande risco para lesões do sistema musculoesquelético ${ }^{7}$.

Considerando que o formato do objeto a ser manuseado pode ser determinante do tipo de preensão utilizada, e que estas tarefas de manuseio estão relacionadas ao aparecimento de lesões musculoesqueléticas, é pertinente caracterizar as diferentes formas de preensão que ocorrem espontaneamente durante o manuseio de objeto do tipo caixa, e possíveis variáveis posturais associadas a esse manuseio. Esse conhecimento pode permitir o estabelecimento de medidas preventivas a serem adotadas em programas ergonômicos de controle de riscos para as lesões musculoesqueléticas em situações ocupacionais.

Assim, este estudo objetivou descrever os diferentes tipos de preensão empregados durante atividade de manuseio (pegar, levantar e abaixar) de cargas de 5 e $10 \mathrm{~kg}$, e quantificar bilateralmente os movimentos de flexo-extensão, desvio radial e ulnar do punho presentes nos manuseios, os quais foram registrados por meio da eletrogoniometria.

\section{MÉTODO}

\section{Sujeitos}

Participaram deste estudo 10 voluntários, do sexo masculino, com idade média 23,3 anos $( \pm 3,16)$ sem treinamento em atividades de manuseio. A estatura média dos indivíduos foi de 176,9 cm $( \pm 4,19)$; e massa corporal média de $74,7 \mathrm{~kg}( \pm 7,11)$. Foram considerados aptos a participar do estudo, os sujeitos que não apresentavam sintomas ou disfunções musculoesqueléticas nos membros superiores.

\section{Materiais e Equipamentos}

Neste estudo foram utilizados: Uma Ficha para caracterização dos voluntários; 3 superfícies de metal de 92 $\mathrm{cm}$ de comprimento por $30 \mathrm{~cm}$ de largura, posicionadas em diferentes alturas a partir do solo, a saber: baixa $(73 \mathrm{~cm})$, intermediária $(103 \mathrm{~cm})$ e alta $(133 \mathrm{~cm})$; uma caixa de metal com tampa removível totalizando 5kg; uma carga extra de metal pesando $5 \mathrm{~kg}$; uma Filmadora Panasonic - modelo ${ }^{\circ}$ GR-AX808U and AA-V35U; um tripé - modelo 950 Tripod; uma balança antropométrica da marca WELMY; e materiais de suporte: fita adesiva simples e dupla face, fita de vídeo, fita métrica, disquetes, tesoura, cinto.

\section{Eletrogoniômetro}

Foi utilizado um conjunto de eletrogoniômetros (Biometrics, Gwent, UK) composto por 1 Data-Logger modelo DL 1001, 2 goniômetros biaxiais - modelo XM 75, 2 cabos de conexão e um computador equipado com software Biometrics V3.X.

\section{Procedimentos}

Os sujeitos foram informados sobre os objetivos e procedimentos do estudo e, concordando em participar, assinaram um termo de consentimento livre e esclarecido. O estudo foi aprovado pelo Comitê de Ética em Pesquisa da Universidade Federal de São Carlos (Protocolo n.059/04). 
Diante da aceitação para participação no estudo, os voluntários foram entrevistados, medidos e pesados e foram feitas as mensurações antropométricas das mãos, segundo critérios de Pheasant ${ }^{8}$. Os sujeitos foram familiarizados com a atividade antes das medidas serem realizadas, entretanto, nenhuma instrução específica sobre como realizar o manuseio, ou pegar a caixa, foi fornecida.

Os procedimentos para a colocação do eletrogoniômetro foram realizados com os sujeitos na posição sentada, com os antebraços pronados sobre uma mesa e as mãos mantidas em posição neutra. Os goniômetros foram fixados, com fita adesiva dupla face, no dorso das mãos e antebraços, alinhados ao tendão extensor do $3^{\circ}$ dedo e eixo maior do antebraço, conforme instruções do fabricante ${ }^{9}$. O equipamento foi então calibrado, convencionando que o canal (A) para a flexo $(+) /$ extensão (-); e o canal (B) para o desvio radial (+) e ulnar do punho direito (-), e os outros dois canais C e D, respectivamente para os movimentos de flexo-extensão e desvio radial e ulnar do punho esquerdo. A taxa de aquisição dos dados foi estabelecida em 1000 amostras/segundo.

\section{Descrição da tarefa}

A tarefa realizada consistiu em pegar a caixa de uma superfície e colocá-la em outra de altura diferente, sendo que a carga sempre deveria partir da altura intermediária e retornar a ela. As superfícies ou alturas de manuseio foram denominadas de Intermediária, Alta, e Baixa. A caixa utilizada para o experimento permitia a alteração de seu peso pela
O movimento registrado pelo eletrogoniômetro foi analisado a partir de gráficos e dos valores das amplitudes em graus, em função do tempo em segundos. A análise possibilitou a identificação do percentual de tempo gasto dentro e fora das faixas de amplitudes pré-estabelecidas. Estas faixas foram escolhidas em função do que a literatura ergonômica ${ }^{6}$ considera como faixas "seguras" de movimento, de forma a evitar desconfortos e sobrecarga ( $\leq 15^{\circ}$ de flexão e extensão, $\leq 10^{\circ}$ de desvio radial, e $\leq 15^{\circ}$ de desvio ulnar).

\section{Análise Estatística}

Uma Análise Univariada - ANOVA foi utilizada para identificar a existência de diferenças significativas na antropometria das mãos dos voluntários. A ANOVA para medidas repetidas foi aplicada para análise da diferenças entre os movimentos empregados para manusear as cargas entre as superfícies. Nesta última análise as duas repetições da seqüência de tarefas foram avaliadas. Para ambas as análises foi utilizado um nível de significância de $\mathrm{p} \leq 0,05$.

\section{RESULTADOS}

As medidas antropometricas das mãos (comprimento das mãos, circunferência metacarpal, circunferência palmar) dos sujeitos foram analisadas, e não mostraram diferenças significativas entre indivíduos ( $\mathrm{p}=0,724)$, e entre as mãos direita e esquerda $(\mathrm{p}=0,821)$.
A

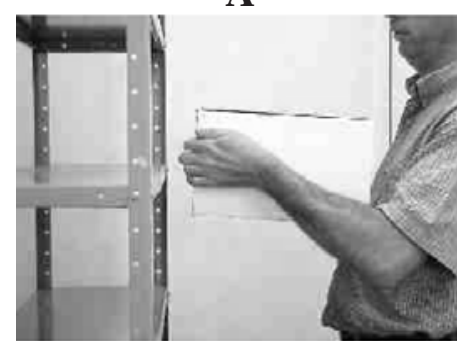

B

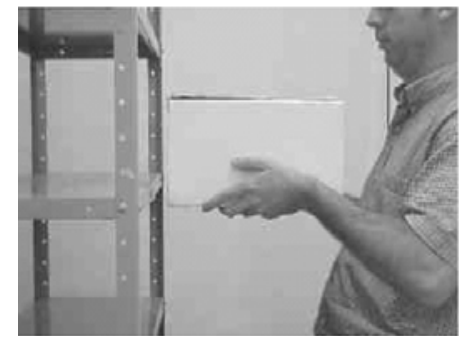

C

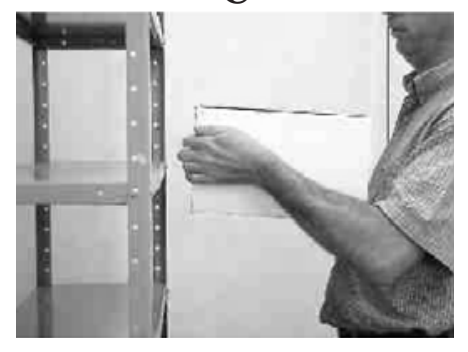

Figura 1. Ilustração das preensões: 1A - Preensão látero-lateral; 1B - Preensão látero-inferior; 1C - Preensão látero-posterior.

introdução de carga interna, assim os sujeitos realizaram uma seqüência com a caixa pesando $5 \mathrm{~kg}$ e outra com $10 \mathrm{~kg}$. Cada seqüência desenvolvida pelos voluntários foi randomizada e repetida duas vezes.

\section{Análise dos Dados}

Os tipos de preensão utilizados durante as atividades de manuseio foram registrados por meio da filmagem, segundo critérios de WATSON ${ }^{10}$, e analisadas descritivamente, o que permitiu a identificação das diferentes categorias de preensão.
Os resultados das filmagens permitiram a identificação de três principais tipos de preensão durante a realização das tarefas, que foram classificadas em látero-lateral, látero-inferior, látero-posterior (Ver Figura1). Os sujeitos não alteravam o tipo de preensão empregado, do início ao término de cada atividade.

A preensão látero-lateral foi definida como sendo aquela em que a mão permaneceu exclusivamente nas laterais da caixa (Figura 1-A).

A preensão látero-inferior foi aquela em que a totalidade (ou maior parte) da mão ficava na lateral da caixa e os 4 dedos 
(do $2^{\circ}$ ao $5^{\circ}$ ) ou parte deles seguravam a parte inferior da caixa (Figura 1-B).

Na preensão látero-posterior os 4 dedos (do $2^{\circ}$ ao $5^{\circ}$ ) ficavam na parte posterior da caixa, com a palma da mão na lateral da caixa e os dedos na face posterior da caixa (Figura 1- C). Pequenas variações foram observadas neste tipo de pega, sendo que às vezes a mão permaneceu deslocada para baixo (próximo ao canto inferior) ou para cima (no canto superior da caixa).

Em termos percentuais o tipo de preensão mais utilizado foi o látero-posterior (38\% das ocorrências), seguida pela látero-inferior, (34\%), e pela látero-lateral (28\%). No que tange a massa da caixa, a preensão látero-lateral foi a mais utilizada quando ocorria manuseio da carga mais leve (5kg). Já as preensões látero-inferiores e látero-posteriores foram mais empregadas com a caixa de $10 \mathrm{~kg}$, sendo que essa tendência ocorreu em todas as alturas de manuseio.

Em média o tempo gasto em cada manuseio foi de 8,77 segundos $( \pm 1,69)$. Esse tempo foi analisado em termos percentuais do tempo total. Os percentuais de tempo gastos pelos sujeitos nos movimentos de flexo/extensão podem ser vistos na Figura 2. Observa-se um predomínio de posturas mais neutras na faixa de $-15^{\circ} / 0^{\circ} /+15^{\circ}$ para os movimentos de flexo/extensão, embora maior tempo foi gasto em flexão nos manuseios envolvendo superfícies altas e maior tempo em extensão nos manuseios envolvendo superfícies baixas.

As posturas neutras também predominaram para os desvios radial e ulnar ver Figura 3, faixa de $-10^{\circ} / 0^{\circ} /+15^{\circ}$. Entretanto, mais de $40 \%$ do tempo, acima de $1 / 3$ do tempo da atividade foi gasto em desvio radial, com amplitudes superiores a 10 graus em manuseios envolvendo superfícies altas, e em torno de $30 \%$ do tempo nessas mesmas condições envolvendo superfície baixa.

A análise estatística permitiu identificar diferenças significativas $(\mathrm{p}=0,000)$ no percentual de tempo gasto nas amplitudes de movimento entre as diferentes alturas de manuseio, contudo não houve diferença significativa entre as cargas manuseadas $(\mathrm{p}=0,43)$.

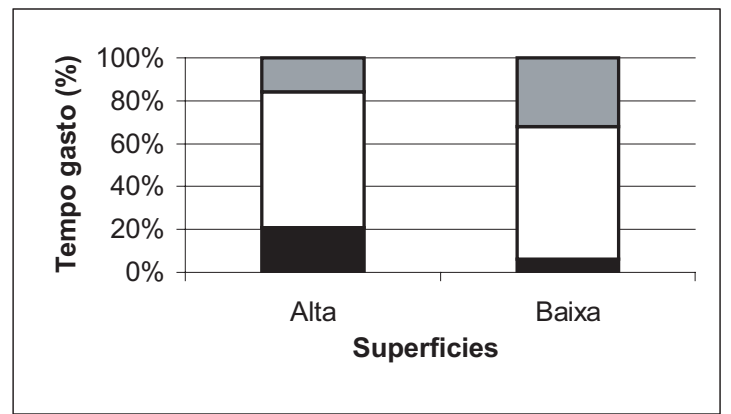

Extensão $>15^{\circ}$

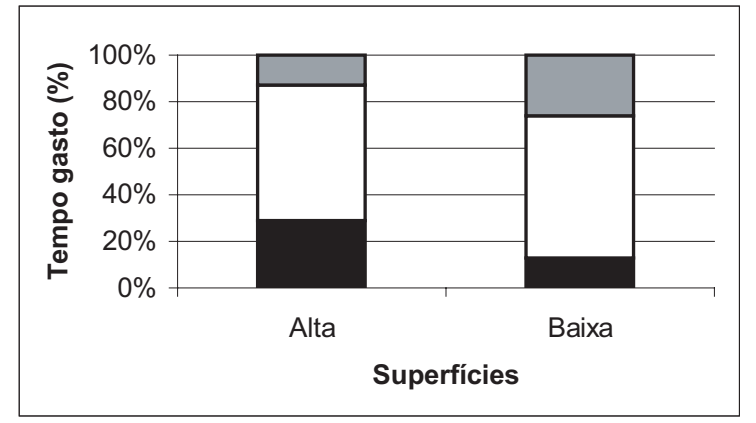

$-15^{\circ} / 0^{\circ}+15^{\circ} \square$ Flexão $>15^{\circ}$

Figura 2. Tempo gasto nas faixas de movimentos de flexo-extensão do punho nas superfícies de manuseio Alta e Baixa para caixas com 5 kg e $10 \mathrm{~kg}$.
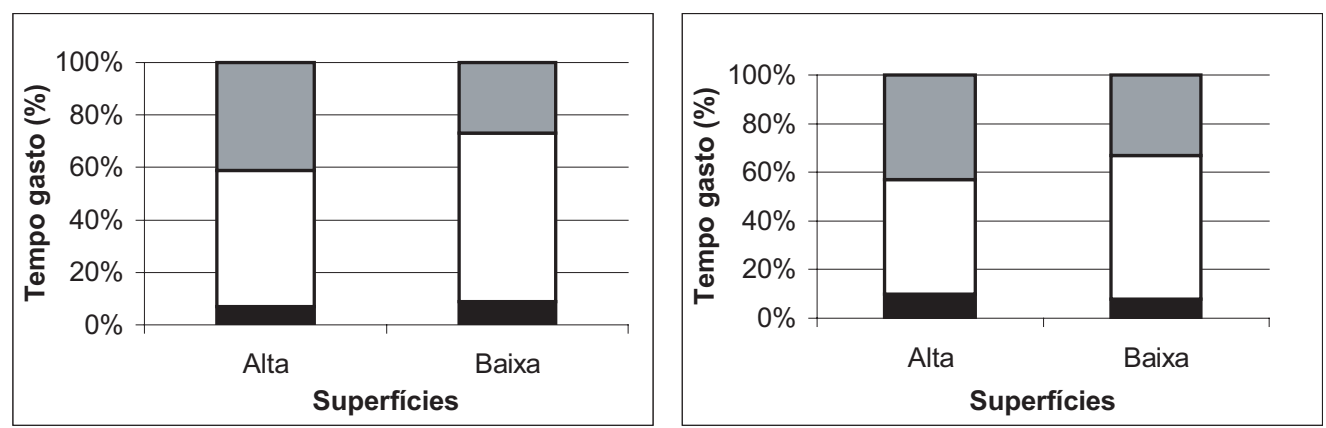

Desvio radial $>10^{\circ}$

$\square-10 \% 0^{\circ}+15^{\circ}$ Desvio ulnar $>15^{\circ}$

Figura 3. Tempo gasto nas faixas de movimentos de desvio radial e ulnar do punho nas superfícies de manuseio Alta e Baixa para caixas com 5 $\mathrm{kg}$ e $10 \mathrm{~kg}$. 


\section{DISCUSSÃO}

Os resultados mostraram que não houve diferenças significativas na antropometria das mãos dos voluntários. Isto indica que diferenças observadas no acoplamento das mãos à caixa não foram influenciadas pela variabilidade individual nas medidas das mãos. Considerou-se pertinente avaliar esse aspecto já que este pode influenciar os tipos de pega e a forma de acoplamento do objeto manuseado ${ }^{11}$.

Os sujeitos optaram por preensões que permitem uma maior estabilidade na sustentação da carga, com predomínio das preensões látero-posteriores e látero-inferiores. $\mathrm{O}$ tipo de preensão escolhido, também permitiu a aproximação da carga ao corpo, diminuindo o braço de alavanca e favorecendo a força. Já a preensão mais utilizada, face inferior da caixa (34\%), pareceu decorrer da necessidade de suportar maior força peso gerada pelo aumento da massa da caixa de 5 para $10 \mathrm{~kg}$.

Estudos realizados por Drury et al. ${ }^{12,13}$, envolvendo manuseio de caixa, mostraram que a posição mais comumente utilizada pelos trabalhadores para esse tipo de tarefa é similar ao que foi denominada nesse estudo de látero-posterior. Isso parecer decorre do fato desta posição fornecer melhor posicionamento das mãos, o que conduz a uma maior estabilidade e melhor controle sobre a carga. Drury ${ }^{12}$ avaliou mais de 2000 tarefas de manuseio em diversas empresas e identificou a utilização de posições assimétricas das mãos em objetos tipo caixa, com um predomínio de pegas onde as mãos estão diagonalmente opostas nos cantos da caixa, posição considerada por ele ideal, pois minimiza as forças envolvidas na preensão.

Baril-Gingras e Lortie ${ }^{14}$, ao estudarem manuseios dinâmicos, encontraram durante a realização das atividades, muitos ajustes na preensão, provavelmente para que seja conseguida uma maior estabilidade na pega; no entanto, os sujeitos analisados no presente estudo mantiveram a mesma postura de preensão do início ao término de cada tarefa, talvez por se tratar de um experimento que envolveu uma atividade relativamente rápida e estática, em que alterações no tipo de preensão empregado eram desnecessárias.

A manutenção do punho em posição neutra é importante para a prevenção de lesões musculoesqueléticas já que amplitudes extremas tensionam ligamentos e comprimem estruturas ${ }^{15}$. $\mathrm{O}$ estudo realizado apontou uma tendência para a utilização de amplitudes neutras, esses resultados podem ter sido influenciados por fatores biomecânicos e da atividade, como a necessidade de ajustes de força e de amplitudes de movimentos, para as diferentes cargas, e o manuseio para diferentes alturas.

As amplitudes de movimento do punho, para os movimentos de desvio ulnar são duas a três vezes mais amplas do que do desvio radial. Além disso, quando as amplitudes de flexão ou extensão do punho são máximas, posição essa que os ligamentos do carpo se encontram alongados, os desvios do punho são mínimos. No entanto, os desvios são máximos se a flexão for mínima ou inexistente, pois esta posição favorece o relaxamento dos ligamentos ${ }^{15}$. Assim, as posturas mais neutras para flexo/extensão podem ter favorecido a permanência de cerca de 35\% do tempo em desvio radial, acima da faixa de amplitude considerada segura $\left(10^{\circ}\right)$. De acordo com Ketola et al. ${ }^{16}$ a amplitude de segurança de 10 o só é válida quando a postura é mantida durante menos de 1/3 do tempo do ciclo da atividade, pois acima dessa proporção à amplitude pode ser considerada de risco. Outra possível explicação para a maior porcentagem de tempo em desvio radial, seria o fato de que quando o movimento ocorria em direção a superfície alta, ou voltando desta superfície, o desvio radial era mais freqüente, talvez para ajustar o eixo da mão ao objeto.

Como os resultados mostraram que os tipos de preensões adotados tiveram relação com as diferentes cargas, seria esperado que as amplitudes dos movimentos realizados pelo punho também apresentassem diferenças significativas em relação às massas; no entanto, isso não foi observado. Deduz-se daí que, como este estudo se ateve apenas à análise dos movimentos do punho (flexão, extensão, desvios radial e ulnar) durante atividade de manuseio de carga (5 e $10 \mathrm{Kg}$ ), possivelmente outras articulações, como a do cotovelo e/ou ombro, sofreram ajustes compensatórios, adotando diferentes posições a fim de compensar a restrição que o formato do objeto propiciava.

Em estudos futuros será importante avaliar as posições articulares de outros segmentos do membro superior, além do punho, durante as atividades de manuseio, e a atividade elétrica de grupos musculares envolvidos na atividade, para detectar outras possíveis condições presentes na preensão de objetos tipo caixa.

\section{CONCLUSÃO}

O estudo mostrou que a massa da caixa manuseada influenciou o tipo de pega adotada, entretanto, não influenciou as amplitudes do punho. As amplitudes de movimentos do punho consideradas seguras predominaram durante as tarefas avaliadas, porém, a altura da superfície, alta ou baixa, influenciou o movimento gerando amplitudes mais extremas. Ainda, uma porcentagem de tempo acima da faixa de amplitude recomendável foi gasta em movimentos de desvio radial quando o manuseio envolvia superfície alta. Assim, programas preventivos deveriam incluir recomendações sobre a estocagem de material a ser manuseado em alturas acessíveis para evitar movimentos mais extremos do punho.

Agradecimentos: Este estudo foi parcialmente apoiado pelo CNPq - Proc. N. 550487/2002-6, e pela Fapesp Proc. N. 04/07207-0. 


\section{REFERÊNCIAS BIBLIOGRÁFICAS}

1. Straker LM. An overview of manual handling injury statistics in western Australia. International Journal of Industrial Ergonomics 1999; 24 (4): 357-8.

2. Ciriello VM, Snook SH, Hashemi L, Cotnam J. Distributions of manual materials handling task parameters. International Journal of Industrial Ergonomics 1999; 23 (4): 379-9.

3. Iida I. Ergonomia Projeto e Produção. São Paulo: Edgard Blücher ; 1992.

4. Putz-Anderson V. Cumulative trauma disorders: a manual for musculoskeletal diseases of the upper limbs. London: Editora Taylor \& Francis; 1988.

5. Mc Gorry RW. A system for the measurement of grip forces and applied moments during hand tool use. Applied Ergonomics 2001; 32: 271-8.

6. Paschoarelli LC, Gil Coury HJC. Amplitudes angulares seguras para avaliação de movimentos da extremidade do membro superior durante atividades: uma revisão. In: XIII Congresso Brasileiro de Ergonomia [CD ROM]. Anais... Fortaleza; 1997. 1 CD-ROM.

7.Campbell SC, Nolan PF, Wharton RK, Train AW. Measurement of forces exerted in the manual handling of small cylindrical objects, Clinical Biomechanics 2000; (25): 349-9.

8. Pheasant S. Anthropometry, Ergonomics and the Design of Work. London: Taylor \& Francis; 1996.

9. Biometrics Ltda. Goniometer and torsiometer operating manual. Gwent, UK; 1997.

10.Watson AWS. Procedure for production of high quality photographs suitable for the recording and evaluation of posture. Revista de Fisioterapia Universidade de São Paulo 1998; 5 (1): 20-6.

11. Paschoarelli LC, Gil Coury HJC. Aspectos Ergonômicos e de Usabilidade no Design de Pegas e Empunhaduras. Estudos em Design 2000; 8 (1): 79-12.

12. Drury CG, Law CH, Pawenski CS. A Survey of Industrial Box Handling: The Human Factors Society 1982; 24 (5): 553-12.

13. Drury CG. The role of the hand in manual materials handling. Ergonomics 1985; 28(1): 213-14.

14. Baril-Gingras G, Lortie M. The handling of objects other than boxes: univariate analysis of handling techniques in a large transport company. Ergonomics 1995; 38(5): 905-20.

15. Kapandji IA. Fisiologia Articular, Volume I: Membro Superior. São Paulo: Manole; 1990.

16. Ketola R, Viikari-Juntura E, Takala E. Validation of a method to assess physical load on the upper extremity. In: Advances in Occupational Ergonomics and Safety 1996. 2 vol, p. 463-4. 